Bull. Austral. Math. Soc.

VOL. 48 (1993) [475-483]

\title{
NONOSCILLATORY SOLUTIONS OF NEUTRAL DELAY DIFFERENTIAL EQUATIONS
}

\author{
Ming-Po Chen, J.S. Yu ANd Z.C. WANg
}

Consider the following neutral delay differential equation

$$
\frac{d}{d t}[x(t)+p x(t-\tau)]+Q(t) x(t-\delta)=0, t \geqslant t_{0}
$$

where $p \in R, \tau \in(0, \infty), \delta \in R^{+}=[0, \infty)$ and $Q \in C\left(\left[t_{0}, \infty\right), R\right)$. We show that if

$$
\int_{0}^{\infty}|Q(s)| d s<\infty
$$

then Equation $\left(^{*}\right)$ has a nonoscillatory solution when $p \neq-1$. We also deal in detail with a conjecture of Chuanxi, Kulenovic and Ladas, and Györi and Ladas.

\section{INTRODUCTION}

Consider the following neutral delay differential equation

$$
\frac{d}{d t}[x(t)+p x(t-\tau)]+Q(t) x(t-\delta)=0, t \geqslant t_{0}
$$

where

$$
p \in R, \tau \in(0, \infty), \delta \in R^{+}=[0, \infty) \text { and } Q \in C\left(\left[t_{0}, \infty\right), R\right) .
$$

Recently, the oscillation and asymptotic behaviour of Equation (1) have been investigated by many authors, see for example $[1,2,3,5,6,7,8]$. For a recent survey, see [4]. All the papers mentioned above, however, assume that $Q(t)$ is nonnegative. Considerably less is known about the behaviour of the solutions of Equation (1) when the coefficient $Q(t)$ is oscillatory. In particular, by combining the result in [5, 7, and 8] we know that if

$$
Q(t) \geqslant 0 \text { and } \int_{t_{0}}^{\infty} Q(s) d s<\infty
$$

Received 21st December, 1992.

This work is partially supported by the NSC of Taipei for the first author, and NNSF of China for the second and third authors.

Copyright Clearance Centre, Inc. Serial-fee code: 0004-9729/93 \$A2.00+0.00. 
then Equation (1) has a nonoscillatory solution when $p \neq-1$. For the critical case $p=-1, Y u$, Wang and Qian [5] found a sufficient condition for the oscillation of all solutions of Equation (1) under the assumption (3). One of our aims in this paper is to study the existence of a nonoscillatory solution of Equation (1) when $Q(t)$ is oscillatory. In section 2 , we show that

$$
\int_{0}^{\infty}|Q(s)| d s<\infty
$$

implies that Equation (1) has a nonoscillatory solution when $p \neq-1$.

In addition, the following result on the asymptotic behaviour of a nonoscillatory solution of Equation (1) has been established by Chuanxi, Kulenovic and Ladas [2], see also [4].

Theorem A. [2] Assume that (2) holds and that

$$
Q(t) \geqslant 0 \text { and } \int_{t_{0}}^{\infty} Q(s) d s=\infty .
$$

Let $x(t)$ be nonoscillatory solution of Equation (1). Then the following statements hold:

(a) If $p<-1$, then $\lim _{t \rightarrow \infty}|x(t)|=\infty$

(b) If $p>-1$, and $p \neq 1$, then $\lim _{t \rightarrow \infty} x(t)=0$.

REMARK 1. As was shown in [1] and [2], the assumption tht $p=-1$, as well as (2) and (5) hold, implies that every solution of Equation (1) oscillates. Thus the assumption in the above Theorem $A$ that $p \neq-1$ is harmless. But, the case $p=1$ has not yet been handled. Therefore, Chuanxi, Kulenovic and Ladas [2] posed the following conjecture. See also [4, Problem 6.12.9 (Conjecture)].

Conjecture B. $[2,4]$ Assume that (2) and (5) hold. Let $x(t)$ be a nonoscillatory solution of the neutral differential equation

$$
\frac{d}{d t}[x(t)+x(t-\tau)]+Q(t) x(t-\delta)=0, t \geqslant t_{0}
$$

Then

$$
\lim _{t \rightarrow \infty} x(t)=0
$$

The second aim in this paper is to answer in detail the above Conjecture B. In section 3, we first give an existence result of a nonoscillatory solution not satisfying (7) of Equation (6), and then by using this result we answer Conjecture B in the negative. 
Finally, we also show that, under appropriate additional hypothesis on $Q(t)$, Conjecture $B$ is also true.

Let $t_{1} \geqslant t_{0}$ and let $\phi \in C\left(\left[t_{1}-m\right], R\right)$, where $m=\max \{\tau, \delta\}$. By a solution of Equation (1) with initial function $\phi$ at $t_{1}$ we mean a function $x \in C\left(\left[t_{1}-m\right], \infty, R\right)$ such that $x(t)=\phi(t)$ for $t \in\left[t_{1}-m, t_{1}\right], x(t)+p x(t-\tau)$ is continuously differentiable for $t \geqslant t_{1}$ and $x(t)$ satisfies Equation (1) for all $t \geqslant t_{1}$.

As usual, a solution of Equation (1) is called nonoscillatory if it is eventually positive or eventually negative and oscillatory if it has arbitrarily large zeros.

\section{NONOSCILLATORY SOLUTIONS OF EQUATION (1)}

In this section we study the existence of a nonoscillatory solution of Equation (1) with $p \neq-1$. The main result in this section is the following theorem.

Theorem 1. Assume that (2) and (4) hold with $p \neq-1$. Then Equation (1) has a nonoscillatory solution.

Proof: The proof of this theorem is rather long and will be divided into five claims. Let $X$ be the set of all continuous and bounded functions on $\left[t_{0}, \infty\right)$ with the sup-norm. Then $X$ is a Banach space.

Cla IM 1. For the case $-1<p \leqslant 0$, choose a $t_{1}>t_{0}$ sufficiently large such that $t_{1}-\tau \geqslant t_{0}, t_{1}-\delta \geqslant t_{0}$ and

$$
\int_{t_{1}}^{\infty}|Q(s)| d s \leqslant \frac{1+p}{4}
$$

Define a bounded, closed and convex subset of $X$ as follows

$$
A=\left\{x \in X ; \frac{2(1+p)}{3} \leqslant x(t) \leqslant \frac{4}{3} \text { for } t \geqslant t_{0}\right\}
$$

Now we define a mapping $T: A \rightarrow X$ as follows

$$
(T x)(t)= \begin{cases}1+p-p x(t-\tau)+\int_{t}^{\infty} Q(s) x(s-\delta) d s, & t \geqslant t_{1} \\ (T x)\left(t_{1}\right), & t_{0} \leqslant t \leqslant t_{1}\end{cases}
$$

Clearly, $T$ is continuous. For every $x \in A$ and $t \geqslant t_{1}$, we see that

and

$$
\begin{aligned}
& (T x)(t) \leqslant 1+p-\frac{4}{3} p+\frac{4}{3} \frac{1+p}{4}=\frac{4}{3} \\
& (T x)(t) \geqslant 1+p-\frac{4}{3} \frac{1+p}{4}=\frac{2(1+p)}{3} .
\end{aligned}
$$


Hence, $(2(1+p)) / 3 \leqslant(T x)(t) \leqslant 4 / 3$ for $t \geqslant t_{0}$ and so $T A \subset A$.

Now we shall shows that $T$ is a contraction mapping on $A$. In fact, for any $x_{1}, x_{2} \in A$ and $t \geqslant t_{1}$ we have

$$
\begin{aligned}
\left|\left(T x_{1}\right)(t)-\left(T x_{2}\right)(t)\right| \leqslant & -p\left|x_{1}(t-\tau)-x_{2}(t-\tau)\right| \\
& \quad+\int_{t}^{\infty}|Q(s)|\left|x_{1}(s-\delta)-x_{2}(s-\delta)\right| d s \\
\leqslant & \frac{1-3 p}{4}\left\|x_{1}-x_{2}\right\| .
\end{aligned}
$$

Then it follows that

$$
\left\|T x_{1}-T x_{2}\right\| \leqslant \frac{1-3 p}{4}\left\|x_{1}-x_{2}\right\| .
$$

Since $0<(1-3 p) / 4<1$, we see that $T$ is a contraction. Therefore, by the Banach contraction principle, $T$ has a fixed point $x \in A$, that is, $T x=x$. Clearly, $x(t)$ is a positive solution of Equation (1) on $\left(t_{1}, \infty\right)$ and so the proof of Claim 1 is complete.

Claim 2. For the case $p<-1$, let $t_{1}>t_{0}$ be such that $t_{1}+\tau-\delta \geqslant t_{0}$ and

Set

$$
\begin{gathered}
\int_{t+\tau}^{\infty}|Q(s)| d s \leqslant-\frac{1+p}{4} . \\
A=\left\{x \in X ;-\frac{p}{2} \leqslant x(t) \leqslant-2 p \text { for } t \geqslant t_{0}\right\} .
\end{gathered}
$$

Then $A$ is a bounded, closed and convex subset of $X$. Define a mapping $T: A \rightarrow X$ as follows

$$
(T x)(t)= \begin{cases}-p-1-\frac{1}{p} x(t+\tau)+\frac{1}{p} \int_{t+\tau}^{\infty} Q(s) x(s-\delta) d s, & t \geqslant t_{1} \\ (T x)\left(t_{1}\right), & t_{0} \leqslant t \leqslant t_{1} .\end{cases}
$$

In is easy to show that $T$ maps $A$ into itself, and by a fashion similar to that in the proof of Claim 1 we see that for any $x_{1}, x_{2} \in A$

$$
\left\|T x_{1}-T x_{2}\right\| \leqslant \frac{p-3}{4 p}\left\|x_{1}-x_{2}\right\| .
$$

This means that $T$ is a contraction, since $0<(p-3) /(4 p)<1$. Therefore, by the Banach contraction principle, $T$ has a fixed point $x \in A$. It is easy to see that this $x$ is a positive solution of Equation (1) and the proof of Claim 2 is finished.

CLAIM 3. For the case $0<p<1$, let $t_{1}>t_{0}$ be such that $t_{1}-\tau \geqslant t_{0}, t_{1}-\delta \geqslant t_{0}$ and

$$
\int_{t_{1}}^{\infty}|Q(s)| d s \leqslant \frac{1-p}{4}
$$

Set

$$
A=\left\{x \in X: 2(1-p) \leqslant x(t) \leqslant 4 \text { for } t \geqslant t_{0}\right\}
$$


which is a bounded, closed and convex subset of $X$. Define $T: A \rightarrow X$ as follows:

$$
(T x)(t)= \begin{cases}3+p-p x(t-\tau)+\int_{t}^{\infty} Q(s) x(s-\delta) d s, & t \geqslant t_{1} \\ (T x)\left(t_{1}\right), & t_{0} \leqslant t \leqslant t_{1} .\end{cases}
$$

It is easy to show that $T$ maps $A$ into $A$ and for any $x_{1}, x_{2} \in A$,

$$
\left\|T x_{1}-T x_{2}\right\| \leqslant \frac{1+3 p}{4}\left\|x_{1}-x_{2}\right\| \text {. }
$$

As $0<(1+3 p) / 4<1$, the Banach contraction principle can be applied to obtain a fixed point $x \in A$. We can see easily that this $x$ is a positive solution of Equation (1). This completes the proof of Claim 3.

ClaIM 4. For the case $p=1$, let $t_{1}>t_{0}$ be such that $t_{1}+\tau-\delta \geqslant t_{0}$ and

$$
\int_{t_{1}+\tau}^{\infty}|Q(s)| d s \leqslant \frac{1}{4}
$$

Clearly, the set

$$
A=\left\{x \in X: 2 \leqslant x(t) \leqslant 4, \text { for } t \geqslant t_{0}\right\}
$$

is a bounded, closed and convex subset of $X$. Define a mapping $T$ on $A$ as follows

$$
(T x)(t)= \begin{cases}3+\sum_{i=1}^{\infty} \int_{t+(2 i-1) r}^{t+2 i \tau} Q(s) x(s-\delta) d s, & t \geqslant t_{1} \\ (T x)\left(t_{1}\right), & t_{0} \leqslant t \leqslant t_{1} .\end{cases}
$$

Clearly $T$ is continuous. It is easy to show that $T$ maps $A$ into $A$ and for any $x_{1}, x_{2} \in A$,

$$
\left\|T x_{1}-T x_{2}\right\| \leqslant \frac{1}{4}\left\|x_{1}-x_{2}\right\| .
$$

Thus, by the Banach contraction principle, $T$ has a fixed point $x \in A$, that is,

$$
x(t)= \begin{cases}3+\sum_{i=1}^{\infty} \int_{t+(2 i-1) \tau}^{t+2 i \tau} Q(s) x(s-\delta) d s, & t \geqslant t_{1}, \\ x\left(t_{1}\right), & t_{0} \leqslant t \leqslant t_{1} .\end{cases}
$$

It follows that $x(t)+x(t-\tau)=6+\int_{t}^{\infty} Q(s) x(s-\delta) d x, t \geqslant t_{1}+\tau$.

From this we see that $x(t)$ is a positive solution of Equation (1) with $p=1$ on $\left[t_{1}+\tau, \infty\right)$ and so the proof for the case $p=1$ is complete.

Claim 5. Finally we consider the last case when $p>1$. Let $t_{1}>t_{0}$ be such that $t_{1}+\tau-\delta \geqslant t_{0}$ and

$$
\int_{t+\tau}^{\infty}|Q(s)| d s \leqslant \frac{p-1}{4}
$$


Consider the bounded, closed and convex subset of $X$;

$$
A=\left\{x \in X: 2(p-1) \leqslant x(t) \leqslant 4 p \text { for } t \geqslant t_{0}\right\},
$$

and define a mapping $T$ on $A$ as follows:

$$
(T x)(t)= \begin{cases}3 p+1-\frac{1}{p} x(t+\tau)+\frac{1}{p} \int_{t+\tau}^{\infty} Q(s) x(s-\delta) d s, & t \geqslant t_{1} \\ (T x)\left(t_{1}\right), & t_{0} \leqslant t \leqslant t_{1} .\end{cases}
$$

By an argument similar to that in the previous four cases, we can easily show that all assumptions of the Banach contraction principle are satisfied. Therefore, $T$ has a fixed point $x \in A$. It is easy to see that this $x$ is a positive solution of Equation (1) on $\left[t_{1}+\tau, \infty\right)$, and the proof of Theorem 1 is complete.

\section{On the Conjecture B}

Consider the neutral delay differential equation

$$
\frac{d}{d t}[x(t)+x(t-\tau)]+Q(t) x(t-\delta)=0
$$

where

$$
\tau>0, \delta \geqslant 0 \text { and } Q \in C\left(\left[t_{0}, \infty\right),(0, \infty)\right) .
$$

First we establish the following result on the existence of a nonoscillatory solution of Equation (8).

THEOREM 2. Assume that (9) holds and there exists a nonnegative continuous function $B(t)$ on $\left[t_{0}, \infty\right)$ such that

$$
B(t)+B(t-\tau)=\text { Constant, } t \geqslant t_{0}+\tau .
$$

Also suppose that there exists a positive number $\lambda$ such that

$$
\lambda\left(1+e^{\lambda \tau}\right) \geqslant Q(t)\left[B(t-\delta) e^{\lambda t}+e^{\lambda \delta}\right], t \geqslant t_{0}+\delta .
$$

Then Equation (8) has a positive solution $x(t)$ satisfying $x(t) \geqslant B(t)$ for $t \geqslant t_{0}+\tau+\delta$ and $x(t)-B(t) \rightarrow 0$ as $t \rightarrow \infty$.

Proof: Set

$$
y(t)=e^{-\lambda t}
$$

Then by (11) we have 


$$
[y(t)+y(t-\tau)]^{\prime}+Q(t)[B(t-\delta)+y(t-\delta)] \leqslant 0, t \geqslant t_{0}+\delta .
$$

Also let

$$
Q^{*}(t)=-[y(t)+y(t-\tau)]^{\prime} /[B(t-\delta)+y(t-\delta)] .
$$

Then $Q^{*}(t) \geqslant Q(t)$ and $y(t)$ satisfies

$$
[y(t)+y(t-\tau)]^{\prime}+Q^{*}(t)[B(t-\delta)+y(t-\delta)]=0, t \geqslant t_{0}+\delta .
$$

From this we have

$$
y(t)+y(t-\tau)=\int_{t}^{\infty} Q^{*}(t)[B(s-\delta)+y(s-\delta)] d s, t \geqslant t_{0}+\delta
$$

which yields

$$
y(t)=\sum_{i=1}^{\infty} \int_{t+(2 i-1) \tau}^{t+2 i \tau} Q^{*}(s)[B(s-\delta)+y(s-\delta)] d s, t \geqslant t_{0}+\delta-\tau
$$

By a slight modification of Lemma 10.5.2 in [4] we can easily see that the corresponding integral equation

$$
z(t)=\sum_{i=1}^{\infty} \int_{t+(2 i-1) \tau}^{t+2 i \tau} Q(s)[B(s-\delta)+z(s-\delta)] d s
$$

has a positive solution $z:\left[t_{0}+\delta-\tau, \infty\right) \rightarrow(0, \infty)$ and $0<z(t) \leqslant y(t)$. From (13) we have

$$
z(t)+z(t-\tau)=\int_{t}^{\infty} Q(s)[B(s-\delta)+z(s-\delta)] d s, t \geqslant t_{0}+\delta .
$$

That is $\quad[z(t)+z(t-\tau)]^{\prime}+Q(t)[B(t-\delta)+z(t-\delta)]=0, t \geqslant t_{0}+\delta$,

which implies that $x(t)=B(t)+z(t)$ is a positive solution of Equation (8) satisfying $x(t)>B(t)$ and $x(t)-B(t) \rightarrow 0$ as $t \rightarrow \infty$. The proof of Theorem 2 is complete.

Note that by using Theorem 2, we can easily construct many examples which show that the answer to Conjective $B$ is negative. In fact for any constant $c>0$, we define

$$
B(t)= \begin{cases}0, & t \in\left[2 i \tau,\left(2 i+\frac{1}{2}\right) \tau\right] \\ \frac{2 c}{\tau}\left[t-\left(2 i+\frac{1}{2}\right) \tau\right], & t \in\left[\left(2 i+\frac{1}{2}\right) \tau,(2 i+1) \tau\right] \\ c, & t \in\left[(2 i+1) \tau,\left(2 i+\frac{3}{2}\right) \tau\right] \\ \frac{2 c}{\tau}[-t+(2 i+1) \tau], & t \in\left[\left(2 i+\frac{3}{2}\right) \tau, 2(i+1) \tau\right]\end{cases}
$$

where $i=0,1,2,3, \ldots$. Clearly

$$
B(t)+B(t-\tau)=c \text { for } t \geqslant \tau
$$


and $B(t)$ is a nonnegative continuous function on $[0, \infty)$. Thus, if $Q(t)$ satisfies for some $\lambda>0$

$$
\frac{\lambda\left(1+e^{\lambda \tau}\right)}{2\left(e^{\lambda \delta}+B(t-\delta) e^{\lambda t}\right)} \leqslant Q(t) \leqslant \frac{\lambda\left(1+e^{\lambda \tau}\right)}{e^{\lambda \delta}+B(t-\delta) e^{\lambda t}}, \text { for } t \geqslant \delta,
$$

then (5) is satisfied and by Theorem 2 Equation (8) has a positive solution $x(t)$ satisfying $x(t)-B(t) \rightarrow 0$ as $t \rightarrow \infty$. But $x(t) \nrightarrow 0$ as $t \rightarrow \infty$, since $\limsup _{t \rightarrow \infty} B(t)=c$.

The above example indeed shows that Conjecture $B$ is not true. But, on the other hand, the following theorem shows that, under appropriate additional hypothesis on $Q(t)$, Conjecture B is true.

Theorem 3. Assume that all the assumptions of Conjecture $B$ hold. Further assume that there exists a positive constant $\beta$ such that $\beta \neq 1$, and

$$
Q(t) \leqslant \beta Q(t-\tau), \text { for } t \geqslant t_{0}+\tau .
$$

Then every nonoscillatory solution of Equation (6) goes to zero as $t \rightarrow \infty$.

Proof: Let $x(t)$ be a nonoscillatory solution of Equation (6). As $-x(t)$ is also a solution of Equation (6), we may assume that $x(t)$ is eventually positive. Thus there exists a $t_{1} \geqslant t_{0}+\tau$ such that $x(t-r)>0, x(t-\delta)>0$ for $t \geqslant t_{1}$. Set

$$
y(t)=x(t)+x(t-\tau) .
$$

Then by (6) we have $\quad y^{\prime}(t) \leqslant 0, y(t)>0$ for $t \geqslant t_{1}$

and

$$
\begin{aligned}
y^{\prime}(t) & =-Q(t) x(t-\delta)=-Q(t) y(t-\delta)+Q(t) x(t-\tau-\delta) \\
& \leqslant-Q(t) y(t-\delta)+\beta Q(t-\tau) x(t-\tau-\delta) \\
& =-Q(t) y(t-\delta)-\beta y^{\prime}(t-\tau) .
\end{aligned}
$$

That is

$$
y^{\prime}(t)+\beta y^{\prime}(t-\tau)+Q(t) y(t-\delta) \leqslant 0 \text { for } t \geqslant t_{1} .
$$

It follows by (5) that

Consequently,

$$
\begin{aligned}
& \lim _{t \rightarrow \infty} y(t)=0 . \\
& \lim _{t \rightarrow \infty} x(t)=0 .
\end{aligned}
$$

The proof of Theorem 3 is complete.

\section{REFERENCES}

[1] Q. Chuanxi and G. Ladas, 'Oscillation of neutral differential equations with variable coefficients', Appl. Anal. 32 (1989), 215-228.

[2] Q. Chuanxi, M.R.S. Kulenovic and G. Ladas, 'Oscillation of neutral equations with variable coefficients', Rad. Mat. 5 (1989), 321-331. 
[3] M.K. Grammatikopoulos, G. Ladas and Y.G. Sficas, 'Oscillation and asymptotic behavior of neutral equations with variable coefficients', Rad. Mat. 2 (1986), 279-303.

[4] I. Györi and G. Ladas, Oscillation theory of delay differential equations with applications (Oxford University Press, 1991).

[5] Jianshe Yu, Zicheng Wang and Chuanxi Qian, 'Oscillation and nonoscillation of neutral delay differential equations', Bull. Austral. Math. Soc. 45 (1992), 195-200.

[6] Zhicheng Wang and Jianshi Yu, 'Oscillation of first order neutral delay differential equations', Kexue Tongbao 35 (1990), 797.

[7] Y. Kitamura and T. Kusano, 'Oscillation and asymptotic behavior of first-order functional differential equations of neutral type', Funkcial. Ekvac. 33 (1990), 325-343.

[8] Jianshe Yu and Zhicheng Wang, 'Nonoscillation of a neutral delay differential equations', Rad. Mat. (to appear).

Institute of Mathematics

Academia Sinica

Nankang, Taipei 11529

Taiwan
Department of Applied Mathematics

Hunan University

Changsha, Hunan 410082

China 